\title{
Contact-Force Control of a Flexible Timoshenko Arm in Rigid/Soft Environment
}

\section{AUTHOR(S):}

Endo, Takahiro; Sasaki, Minoru; Matsuno, Fumitoshi; Jia, Yingmin

\section{CITATION:}

Endo, Takahiro ... [et al]. Contact-Force Control of a Flexible Timoshenko Arm in Rigid/Soft Environment. IEEE Transactions on Automatic Control 2017, 62(5): 2546-2553

\section{ISSUE DATE:}

2017-05

URL:

http://hdl.handle.net/2433/232624

\section{RIGHT:}

(C) 2017 IEEE. Personal use of this material is permitted. Permission from IEEE must be obtained for all other uses, in any current or future media, including reprinting/republishing this material for advertising or promotional purposes,

creating new collective works, for resale or redistribution to servers or lists, or reuse of any copyrighted component of this work in other works.; This is not the published version. Please cite only the published version.; この論文は出版社版

でありません。引用の際には出版社版をご確認ご利用ください。 


\title{
Contact-Force Control of a Flexible Timoshenko Arm in Rigid/Soft Environment
}

\author{
Takahiro Endo, Minoru Sasaki, Fumitoshi Matsuno, and Yingmin Jia
}

\begin{abstract}
This paper discusses a contact-force control problem of a one-link flexible arm. This flexible arm includes a Timoshenko beam, and thus we call it the flexible Timoshenko arm. The primary aim is to control the contact force at the contact point. To do so, we first apply our previously proposed force controller, which exponentially stabilizes the closed-loop system of a flexible Euler-Bernoulli arm, to the force-control problem of the flexible Timoshenko arm. We then show that our previously proposed force controller cannot exponentially stabilize the flexible Timoshenko arm. Next, we consider the flexible Timoshenko arm, which is making contact with a soft environment. By utilizing the damping force in the soft environment, as well as the controller, we try to overcome the problem. We then prove the exponential stability of the closed-loop system. Finally, we provide simulation results, and consider the validity of our force controller.
\end{abstract}

Index Terms-Distributed parameter systems, Flexible arm, Timoshenko beam, Force control, Stability.

\section{INTRODUCTION}

A flexible arm is a robotic arm with elastic links. The dynamics of the elastic links are expressed by partial differential equations (PDEs), and the dynamics of the actuators, tip load, and others are expressed by ordinary differential equations (ODEs). Thus, the flexible arm is a hybrid PDE-ODE system. For the dynamics of flexible arms, the Timoshenko beam is widely used to represent the dynamics of the elastic links, and we describe such arms as flexible Timoshenko arms. There have been several relevant previous studies of the flexible Timoshenko arm as a hybrid PDE-ODE system [1]-[8].

These studies mainly focused on vibrations control which is generally insufficient to enable the flexible arm to be used for complex tasks. It is also important to control the contact force that the end-effector of the flexible arm exerts on an object or the environment [9], [10]. In this paper, we focus on a force control problem of a one-link flexible Timoshenko arm in infinite dimensional settings.

There have been a few studies of the contact-force control of a flexible arm based on the infinite dimensional model [11]-[18]. These studies [11]-[15] considered the force-control problem for a one-link flexible arm modeled by an Euler-Bernoulli beam, and asymptotic/exponential stabilizing controllers were proposed. On the other hand, studies have also discussed cooperative tasks [16]-[18], which are the typical

This work was supported in part by JSPS KAKENHI Grant Number 23700143

T. Endo and F. Matsuno are with the Department of Mechanical Engineering and Science, Kyoto University, Kyoto, Japan (e-mail endo@me.kyoto-u.ac.jp, matsuno@me.kyoto-u.ac.jp).

M. Sasaki is with the Department of Mechanical Engineering, Gifu University, Gifu, Japan (e-mail: sasaki@gifu-u.ac.jp).

Y. Jia is with the School of Automation Science and Electrical Engineering, Beihang University, Beijing, China (e-mail: ymjia@buaa.edu.cn). tasks of force control, by multiple flexible arms also modeled by Euler-Bernoulli beams. However, to the best of our knowledge, there has not yet been a research conducted on force control for a flexible Timoshenko arm based on the infinite dimensional model. The Timoshenko beam includes the effects of shear and rotation in the Euler-Bernoulli beam, and thus is a modified model for a non-slender beam and high-frequency response. Therefore, the Timoshenko beam can be used in a wider range of applications than the Euler-Bernoulli beam [19], [20]. The contact-force control problem of the flexible Timoshenko arm is a challenging and important problem, and thus we deal with it in this paper.

First, we apply our previously proposed force controller [14], [17] to the force-control problem of the flexible Timoshenko arm making contact with a rigid environment. Here, our controller was proposed for force-control of a flexible Euler-Bernoulli arm as it made contact with a rigid environment, and the controller exponentially stabilized the closed-loop system. Using this controller, we show that force control of the flexible Timoshenko arm is realized. We note here that there is a controller that exponentially stabilizes the Euler-Bernoulli beam but destabilizes the Timoshenko beam in the case of the vibration-suppression problem (this was not the contact-force control problem; also, in that case, the system was not modeled by the hybrid PDE-ODE but by the PDE model) [21]. However, the controller in this paper does not create such a problem. In addition, we show that our previous controller asymptotically stabilizes the closed-loop system of the flexible Timoshenko arm but cannot exponentially stabilize it.

To overcome this problem, we consider the contact-force control problem of the flexible Timoshenko arm making contact with a soft environment. By utilizing the damping force of the soft environment, we prove the exponential stability of the closed-loop system using the frequency domain method. From a practical point of view, there are many situations in which the environment is soft rather than rigid, and thus the contact-force control problem in a soft environment is an important one.

This paper is organized as follows. In Section II, we describe the mathematical model of a one-link flexible Timoshenko arm constrained to a rigid environment. Further, we formulate the contact-force control problem, and introduce our previously proposed force controller. Section III describes the semigroup setting of the system, as well as its asymptotic stability and non-exponential stability. We improve the mathematical model to that of a one-link flexible Timoshenko arm making contact with a soft object, and discuss its semigroup setting in Section IV, then prove its exponential stability in Section V. The simulation results are shown in Section VI. Finally, Section VII presents our conclusions. 


\section{DESCRIPTION OF THE PROBLEM}

\section{A. Dynamics of a constrained flexible Timoshenko arm}

Fig. 1 shows a constrained one-link flexible Timoshenko arm. One end of the arm is fixed to the rotational motor. The other end makes contact with the surface of a rigid object, and thus the arm has a pinned boundary at the tip, as shown in [22]. The flexible arm rotates in the horizontal plane, and is not affected by the acceleration of gravity. With length $l$, mass density $\rho$, cross sectional area $A$, area moment of inertia $I$, Young's modulus $E$, shear modulus $G$, and shear coefficient $\kappa$, the flexible arm satisfies the Timoshenko beam hypothesis.

In Fig. 1, $X Y$ is an absolute coordinate system and $x y$ is a local coordinate system. In addition, $x y$ rotates with the motor. Let $J, \tau_{m}(t)$, and $\theta(t)$ be the inertia moment, torque, and rotational angle of the motor, respectively. Further, let $w(x, t)$ and $\phi(x, t)$ be the transverse displacement of the flexible arm at time $t$ and spatial point $x \in(0, l)$, and the rotation of the cross section due to bending deformation, respectively. Note that $w(x, t), \phi(x, t)$, and $\theta(t)$ are assumed to be small.

The boundary at the tip makes contact with the surface of the rigid object, and thus we obtain the following geometric constraint: $\Lambda \equiv l \theta(t)+w(l, t)=0$. The kinetic energy $E_{k}$ and the potential energy $E_{p}$ of the overall system are given by the following: $2 E_{K}=\rho_{1} \int_{0}^{l}[x \dot{\theta}(t)+\dot{w}(x, t)]^{2} \mathrm{~d} x+\rho_{2} \int_{0}^{l}[\dot{\theta}(t)+\dot{\phi}(x, t)]^{2} \mathrm{~d} x+$ $J \dot{\theta}^{2}(t), 2 E_{P}=E I \int_{0}^{l}\left[\phi^{\prime}(x, t)\right]^{2} \mathrm{~d} x+K \int_{0}^{l}\left[\phi(x, t)-w^{\prime}(x, t)\right]^{2} \mathrm{~d} x$, where, $\rho_{1}=\rho A, \rho_{2}=\rho I, K=\kappa G A$, a dot denotes the time derivative, and a prime denotes the partial derivative with respect to $x$. Here the virtual work is given by $\delta W=\tau_{m}(t) \delta \theta(t)$.

Now we can obtain the following equations of motion by applying Hamilton's principle and the Lagrange multiplier, and using the procedure described in [14]: for $x \in(0, l)$ and $t \geq 0$

$$
\begin{aligned}
& \rho_{1}[\ddot{w}(x, t)+x \ddot{\theta}(t)]+K\left[\phi^{\prime}(x, t)-w^{\prime \prime}(x, t)\right]=0, \\
& \rho_{2}[\ddot{\phi}(x, t)+\ddot{\theta}(t)]+K\left[\phi(x, t)-w^{\prime}(x, t)\right]-E I \phi^{\prime \prime}(x, t)=0, \\
& w(0, t)=\phi(0, t)=\phi^{\prime}(l, t)=l \theta(t)+w(l, t)=0, \\
& J \ddot{\theta}(t)=\tau_{m}(t)+E I \phi^{\prime}(0, t) \equiv \tau(t),
\end{aligned}
$$

with the algebraic relation

$$
\lambda(t)=K\left[w^{\prime}(l, t)-\phi(l, t)\right],
$$

where $\lambda(t)$ is the Lagrange multiplier and is equivalent to the contact force, which arises in the direction along the normal vector of the constraint surface.

\section{B. Control objective}

To control the contact-force at the tip of the arm, we set the

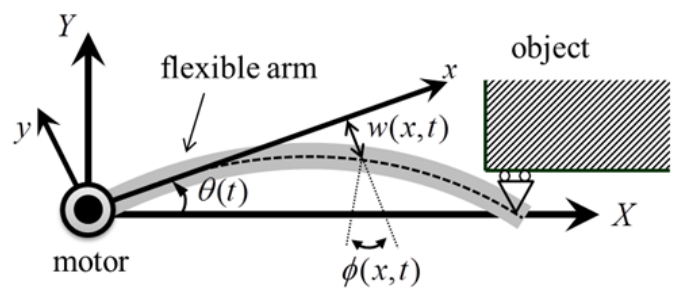

Fig. 1. Flexible Timoshenko arm making contact with a rigid object. control objective as follows: to construct a controller satisfying $\lambda(t) \rightarrow \lambda_{d}, \dot{w}(x, t) \rightarrow 0, \dot{\phi}(x, t) \rightarrow 0, \dot{\theta}(t) \rightarrow 0$, as $t \rightarrow \infty$ where $\lambda_{d}$ is the constant desired contact force. At the desired equilibrium point $\left(\lambda(t)=\lambda_{d}, \dot{w}(x, t)=\dot{\phi}(x, t)=\dot{\theta}(t)=0\right), w(x, t)$ and $\phi(x, t)$ become functions of the variable $x$, and $\theta(t)$ becomes constant. Thus, we describe them as $w_{d}(x), \phi_{d}(x)$, and $\theta_{d}$, respectively. By substituting these into (1)-(5), we obtain:

$$
\left\{\begin{array}{l}
w_{d}(x)=\lambda_{d} x\left(\frac{1}{K}+\frac{l x}{2 E I}-\frac{x^{2}}{6 E I}\right), \quad \phi_{d}(x)=\frac{\lambda_{d} x}{E I}\left(l-\frac{x}{2}\right), \\
\theta_{d}=-\lambda_{d}\left(\frac{1}{K}+\frac{l^{2}}{3 E I}\right),
\end{array}\right.
$$

where, $w_{d}(x), \phi_{d}(x)$, and $\theta_{d}$ mean a static transverse displacement, a static rotation of the cross section, and a static angle of the motor in the case where the contact force converges to the desired value, respectively. Here, we see that $w_{d}(x)$ and $\phi_{d}(x)$ are coupled through $\lambda_{d}$. In addition, we see that we cannot set $\lambda_{d}$ and $\theta_{d}$ independently.

Based on these considerations, we consider a force controller that realizes the following:

$$
\left\{\begin{array}{l}
w(x, t) \rightarrow w_{d}(x), \dot{w}(x, t) \rightarrow 0, \\
\phi(x, t) \rightarrow \phi_{d}(x), \dot{\phi}(x, t) \rightarrow 0, \theta(t) \rightarrow \theta_{d}, \dot{\theta}(t) \rightarrow 0 .
\end{array} \text { as } t \rightarrow \infty\right.
$$

\section{Our previously proposed force controller}

To achieve the objectives stated in (8), first we use our previously proposed force controller [14], [17]

$$
\tau(t)=\widetilde{k}_{1} E I\left[\phi^{\prime}(0, t)-\phi_{d}^{\prime}(0)\right]+\widetilde{k}_{2} E I \dot{\phi}^{\prime}(0, t)-\widetilde{k}_{3}\left[\theta(t)-\theta_{d}\right]-\widetilde{k}_{4} \dot{\theta}(t),
$$

where the feedback gain $\widetilde{k}_{i}, i=1, \ldots, 4$, is a positive constant. This controller was proposed for the contact-force control of a flexible Euler-Bernoulli arm with a rigid environment. Here, we use this controller for the contact-force control of the flexible Timoshenko arm. In this controller, the first and second terms are for $\phi(x, t) \rightarrow \phi_{d}(x)$ and $\dot{\phi}(x, t) \rightarrow 0$, and the third and the fourth terms are for $\theta(t) \rightarrow \theta_{d}$ and $\dot{\theta}(t) \rightarrow 0$. Here, if $\phi(x, t)$ $\rightarrow \phi_{d}(x)$ is satisfied in the steady state, then $w(x, t)=w_{d}(x)$ and $\lambda(t)=\lambda_{d}$ are satisfied. Thus, the controller (9) has the potential to realize (8). In previous studies, the controller in [14] had no third and fourth terms, while the controller used in [17] did have the third and the fourth terms. In the case where the controlled system is the flexible Timoshenko arm, we need to introduce the third and the fourth terms for the state space $H$, defined in (16), to become a Hilbert space under the inner product (17).

\section{CLOSED-LOOP SYSTEM AND ITS STABILITY}

\section{A. Semigroup setting}

Now let us introduce new variables

$$
\left\{\begin{array}{l}
y_{1}(x, t)=\left[w(x, t)-w_{d}(x)\right]+x\left[\theta(t)-\theta_{d}\right], \\
y_{2}(x, t)=\left[\phi(x, t)-\phi_{d}(x)\right]+\left[\theta(t)-\theta_{d}\right], \\
\eta(t)=k_{2} E I y_{2}^{\prime}(0, t)-\dot{y}_{2}(0, t)-k_{2} k_{3} y_{2}(0, t) / k_{1},
\end{array}\right.
$$

based on the procedure described in [14], [17]. Then, the equations of motion become simple, the equilibrium point is 
moved to its origin, and the closed-loop system can be rewritten as follows: for $x \in(0, l)$ and $t \geq 0$

$$
\begin{aligned}
& \rho_{1} \ddot{y}_{1}(x, t)+K\left[y_{2}^{\prime}(x, t)-y_{1}^{\prime \prime}(x, t)\right]=0, \\
& \rho_{2} \ddot{y}_{2}(x, t)+K\left[y_{2}(x, t)-y_{1}^{\prime}(x, t)\right]-E I y_{2}^{\prime \prime}(x, t)=0, \\
& y_{1}(0, t)=y_{1}(l, t)=y_{2}^{\prime}(l, t)=0, \\
& \dot{\eta}(t)=-k_{1} E I y_{2}^{\prime}(0, t)+k_{3} y_{2}(0, t)+D \dot{y}_{2}(0, t),
\end{aligned}
$$

with the algebraic relation

$$
\lambda(t)-\lambda_{d}=K\left[y_{1}^{\prime}(l, t)-y_{2}(l, t)\right] \text {, }
$$

where $k_{i}=\tilde{k}_{i} / J$ for $i=1, \ldots, 4$, and $D=k_{4}-k_{2} k_{3} / k_{1}$.

To formulate the closed-loop system in an appropriate Hilbert space, let us introduce the following Hilbert space:

$$
H=H_{0}^{1}(0, l) \times L^{2}(0, l) \times H^{1}(0, l) \times L^{2}(0, l) \times \mathrm{C},
$$

where the space $H^{m}(0, l)$ is the usual Sobolev space of order $m$, $L^{2}(0, l)$ is the usual square integrable functional space, $H_{0}^{m}(0, l)=\left\{u \in H^{m}: u(0)=u(l)=0\right\}$, and $\mathrm{C}$ is the set of complex numbers. In space $H$, we define the inner product as follows:

$$
\begin{aligned}
& \langle z, \hat{z}\rangle_{H}=\frac{\rho_{1}}{2} \int_{0}^{l} v_{1} \overline{\hat{v}_{1}} \mathrm{~d} x+\frac{\rho_{2}}{2} \int_{0}^{l} v_{2} \overline{\hat{v}_{2}} \mathrm{~d} x+\frac{E I}{2} \int_{0}^{l} u_{2}^{\prime} \overline{\hat{u}_{2}^{\prime}} \mathrm{d} x \\
& \quad+\frac{K}{2} \int_{0}^{l}\left(u_{2}-u_{1}^{\prime}\right) \overline{\left(\hat{u}_{2}-\hat{u}_{1}^{\prime}\right)} \mathrm{d} x+\frac{k_{3}}{2 k_{1}} u_{2}(0) \overline{\hat{u}_{2}(0)}+\frac{1}{2\left(k_{1}+k_{2} D\right)} \eta \overline{\hat{\eta}},
\end{aligned}
$$

for $z=\left(u_{1}, v_{1}, u_{2}, v_{2}, \eta\right)^{T}$, and $\hat{z}=\left(\hat{u}_{1}, \hat{v}_{1}, \hat{u}_{2}, \hat{v}_{2}, \hat{\eta}\right)^{T} \in H$. Here we assumed that $k_{1}+k_{2} D>0$, which holds if $D>0$. It can be shown that $H$, together with (17), becomes a Hilbert space. In addition, we also define a linear operator $A: D(A) \subset H \rightarrow H$ by

$$
\begin{array}{r}
A z=\left[v_{1}, \frac{K}{\rho_{1}}\left(u_{1}^{\prime \prime}-u_{2}^{\prime}\right), v_{2}, \frac{K}{\rho_{2}}\left(u_{1}^{\prime}-u_{2}\right)+\frac{E I}{\rho_{2}} u_{2}^{\prime \prime},\right. \\
\left.-k_{1} E I u_{2}^{\prime}(0)+k_{3} u_{2}(0)+D v_{2}(0)\right]^{T},
\end{array}
$$

with domain

$$
\begin{aligned}
D(A)=\left\{\begin{array}{l}
z \in H_{0}^{2}(0, l) \times H_{0}^{1}(0, l) \times H^{2}(0, l) \times H^{1}(0, l) \times \mathrm{C}: \\
\left.u_{2}^{\prime}(l)=0, \eta=k_{2} E I u_{2}^{\prime}(0)-v_{2}(0)-k_{2} k_{3} u_{2}(0) / k_{1}\right\} .
\end{array}\right.
\end{aligned}
$$

The closed-loop system (11)-(14) can then be written as the following first order evolution equation on $H$ :

$\dot{z}(t)=A z(t), \quad z(0)=z_{0}$,

where $z(t)=\left(y_{1}(\cdot, t), \dot{y}_{1}(\cdot, t), y_{2}(\cdot, t), \dot{y}_{2}(\cdot, t), \eta(t)\right)^{T}$ is the state, and $z_{0}$ is the initial value.

\section{B. Properties of the closed-loop system}

We obtain the following lemmas for the properties of the closed-loop system:

Lemma 1: If the feedback gain $k_{i}, i=1, \ldots, 4$, satisfies

$k_{1} k_{4}>k_{2} k_{3}$,

that is, if $D>0$, then the operator $A$ generates a $C_{0}$-semigroup of contractions. In addition, the operator $A^{-1}$ is compact. Therefore, the spectrum $\sigma(A)$ of the operator $A$ consists only of the isolated eigenvalues.

Proof: First, we show that the operator $A$ is dissipative. For any $z=\left(u_{1}, v_{1}, u_{2}, v_{2}, \eta\right)^{T} \in D(A)$, we obtain

$$
\begin{aligned}
& 2 \operatorname{Re}\langle A z, z\rangle_{H} \\
& =-\frac{k_{2}}{k_{1}\left(k_{1}+k_{2} D\right)}\left|k_{1} E I u_{2}^{\prime}(0)-k_{3} u_{2}(0)\right|^{2}-\frac{D}{k_{1}+k_{2} D}\left|v_{2}(0)\right|^{2} \leq 0 .
\end{aligned}
$$

This means that the operator $A$ is dissipative.

Next, we show that $0 \in \rho(A)$, where $\rho(A)$ is the resolvent set of the operator $A$. For any given $\hat{z}=\left(\hat{u}_{1}, \hat{v}_{1}, \hat{u}_{2}, \hat{v}_{2}, \hat{\eta}\right)^{T} \in H$, we seek a solution $z=\left(u_{1}, v_{1}, u_{2}, v_{2}, \eta\right)^{T} \in D(A)$ of $-A z=\hat{z}$. Eliminating $v_{1}$, $v_{2}$, and $\eta$ in this equation leads to the following equations:

$$
\left\{\begin{array}{l}
K\left[u_{2}^{\prime}(x)-u_{1}^{\prime \prime}(x)\right]=\rho_{1} \hat{v}_{1}(x), \\
K\left[u_{2}(x)-u_{1}^{\prime}(x)\right]-E I u_{2}^{\prime \prime}(x)=\rho_{2} \hat{v}_{2}(x), \\
u_{1}(0)=u_{1}(l)=u_{2}^{\prime}(l)=0, \quad k_{1} E I u_{2}^{\prime}(0)-k_{3} u_{2}(0)=\hat{\eta}-D \hat{u}_{2}(0) .
\end{array}\right.
$$

Now, we integrate the first equation of (23) and substitute it into the second equation of (23). The obtained equation then yields $\quad u_{2}(x)=\rho_{1} /(2 E I) \int_{0}^{x}(x-s)^{2} \hat{v}_{1}(s) \mathrm{d} s-\rho_{2} /(E I) \int_{0}^{x}(x-s) \hat{v}_{2}(s) \mathrm{d} s+$ $x^{2} C_{1} /(2 E I)+C_{2} x+C_{3}$, where $C_{i}, i=1,2,3$, is a constant, which is to be determined by the boundary conditions. Further, from the first equation of (23) and $u_{2}$, we obtain $u_{1}(x)=$ $\rho_{1} \int_{0}^{x} \alpha[x-s] \hat{v}_{1}(s) \mathrm{d} s-\rho_{2} /(2 E I) \int_{0}^{x}(x-s)^{2} \hat{v}_{2}(s) \mathrm{d} s+\alpha[x] C_{1}+x^{2} C_{2} / 2+C_{3} x$, where $\alpha[x] \equiv x^{3} /(6 E I)-x / K$. Substituting these solutions into the remaining boundary conditions, we get the matrix form relation: $M\left[C_{1}, C_{2}, C_{3}\right]^{T}=\left[f_{1}, f_{2}, f_{3}\right]^{T}$, where $M \in \mathrm{C}^{3 \times 3}$ is a matrix and $f_{i}, i=1,2,3$, is a scalar. A straightforward calculation shows $\operatorname{det} M=1 /\left(k_{3} l / K+k_{1} l^{2}+k_{3} l^{3} /(3 E I)\right) \neq 0$, and thus the coefficient $C_{i}, i=1,2,3$, can be uniquely determined. The remaining unknowns $v_{1}, v_{2}$, and $\eta$ can be found using $u_{1}$ and $u_{2}$. Therefore, we obtain $0 \in \rho(A)$.

As the operator $A$ is dissipative and $0 \in \rho(A)$, we conclude that the operator $A$ generates a $C_{0}$-semigroup of contractions [23].

Finally, the compactness of the operator $A^{-1}$ is a direct consequence of Sobolev imbedding [24].

Let $T(t)$ be a $C_{0}$-semigroup of contractions generated by the operator $A$. Then, Lemma 1 means that the closed-loop system (20) has a unique solution $z(t)=T(t) z_{0} \in D(A)$, for $z_{0} \in D(A)$. Further, $y_{1}$ and $y_{2} \in H^{2}(0, l)$ in the solution, and thus the contact force $\lambda(t)-\lambda_{d}=K\left[y_{1}^{\prime}(l, t)-y_{2}(l, t)\right]$ also exists.

Lemma 2: We assume that (21) holds. Then, there are no eigenvalues on the imaginary axis.

Proof: Let $s \in i \mathrm{R}, s \neq 0$, and $\varphi=\left(\varphi_{1}, \varphi_{2}, \varphi_{3}, \varphi_{4}, \varphi_{5}\right)^{T} \in D(A)$ be an eigenvalue and the corresponding eigenfunction of the operator $A$, respectively. Note here that we have shown that zero is not an eigenvalue, in Lemma 1. Then, we obtain $\operatorname{Re}\langle A \varphi, \varphi\rangle_{H}=0$, which, together with (22) give $\varphi_{4}(0)=0$ and $k_{1} E I \varphi_{3}^{\prime}(0)-k_{3} \varphi_{3}(0)=0$. Based on these relations, let us consider the eigenvalue problem $A \varphi=s \varphi$. Eliminating $\varphi_{2}, \varphi_{4}$, and $\varphi_{5}$, 
we obtain the following equations:

$\left\{\begin{array}{l}K\left(\varphi_{1}^{\prime \prime}-\varphi_{3}^{\prime}\right)=\rho_{1} s^{2} \varphi_{1}, \quad K\left(\varphi_{1}^{\prime}-\varphi_{3}\right)+E I \varphi_{3}^{\prime \prime}=\rho_{2} s^{2} \varphi_{3}, \\ \varphi_{1}(0)=\varphi_{3}(0)=\varphi_{3}^{\prime}(0)=0, \quad \varphi_{1}(l)=\varphi_{3}^{\prime}(l)=0 .\end{array}\right.$

The first three equations in (24) give

$$
\left\{\begin{aligned}
& \varphi_{1}(x)=\frac{c_{1}}{\sqrt{\mu_{1} \mu_{2}}\left(\mu_{1}-\mu_{2}\right)}\left[\left(b-\mu_{1}\right) \sqrt{\mu_{2}} \sinh \sqrt{\mu_{1}} x\right. \\
&\left.-\left(b-\mu_{2}\right) \sqrt{\mu_{1}} \sinh \sqrt{\mu_{2}} x\right], \\
& \varphi_{3}(x)=\frac{c c_{1}}{\mu_{1}-\mu_{2}}\left[\cosh \sqrt{\mu_{1}} x-\cosh \sqrt{\mu_{2}} x\right],
\end{aligned}\right.
$$

where $a=\rho_{1} s^{2} / K, b=\left(\rho_{2} s^{2}+K\right) / E I$, and $c=-K / E I$. In addition, $\mu_{1}$ and $\mu_{2}$ are roots of the polynomial $\mu^{2}-(a+b+c) \mu+a b=0$, and we can show that $\mu_{1} \neq \mu_{2}$ using the same procedure described in [25]. The constant $c_{1}$ is determined by the remaining boundary conditions. Now, the functions given by (25) together with the boundary condition $\varphi_{3}^{\prime}(l)=0$ and the fact $\mu_{1} \neq \mu_{2}$ lead to $\varphi_{1}(x)=\varphi_{3}(x)=0$. This means $\varphi=0$, which contradicts the fact that $\phi$ is an eigenfunction. Therefore, there are no eigenvalues on the imaginary axis.

For the asymptotic stability of the closed-loop system, we summarize the following theorem:

Theorem 1: If the feedback gain $k_{i}, i=1, \ldots, 4$, satisfies (21), then the closed-loop system (20) is asymptotically stable.

Proof: From Lemma 1 and 2, we can apply the Arendt-Batty theorem [26] to our system, which concludes that the closed-loop system (20) is asymptotically stable.

\section{Non-exponential stability of the closed-loop system}

Next, we consider the spectrum analysis and show that the closed-loop system cannot be exponentially stable. Let us consider the eigenvalue problem $A \varphi=s \varphi$, where $s \in \mathrm{C}$ is an eigenvalue of the operator $A$, and $\varphi=\left(\varphi_{1}, \varphi_{2}, \varphi_{3}, \varphi_{4}, \varphi_{5}\right)^{T} \in D(A)$ is the eigenfunction. Eliminating $\varphi_{2}, \varphi_{4}$, and $\varphi_{5}$, we obtain the following equations:

$$
\left\{\begin{array}{l}
\varphi_{1}^{\prime \prime}(x)=\varphi_{3}^{\prime}(x)+\gamma_{1}^{2} s^{2} \varphi_{1}(x), \\
\varphi_{3}^{\prime \prime}(x)=\gamma_{2}^{2} s^{2} \varphi_{3}(x)+d\left[\varphi_{3}(x)-\varphi_{1}^{\prime}(x)\right], \\
\varphi_{1}(0)=\varphi_{1}(l)=\varphi_{3}^{\prime}(l)=0 \\
\left(k_{2} s+k_{1}\right) E I \varphi_{3}^{\prime}(0)-\left(s^{2}+k_{4} s+k_{3}\right) \varphi_{3}(0)=0,
\end{array}\right.
$$

where $\gamma_{1}^{2}=\rho_{1} / K, \gamma_{2}^{2}=\rho_{2} / E I$, and $d=K / E I$.

Here, we are interested in whether the closed-loop system is exponentially stable. We thus study the asymptotic analysis of the eigenvalues with large moduli in the strip $\Pi=\{s \in \mathrm{C}$ : $-\alpha \leq \operatorname{Re}(s) \leq 0\}$ for some $\alpha>0$, as was the case in [27]. For the eigenvalues with large moduli, we obtain the following lemma.

Lemma 3: We assume that $\gamma_{2} k_{2} E I \neq 1$ and $\gamma_{1} \neq \gamma_{2}$. Then, the eigenvalues $s_{n}, n=1,2, \cdots$, of the operator $A$ with large moduli in $\Pi$ have the following two asymptotic branches:

$$
\begin{aligned}
& s_{1 n}=\frac{n \pi}{\gamma_{1} l} i+O\left(n^{-1}\right), \\
& s_{n}=\left\{\begin{array}{l}
\frac{1}{2 \gamma_{2} l}\left[\ln \left|\frac{\gamma_{2} k_{2} E I-1}{\gamma_{2} k_{2} E I+1}\right|+2 n \pi i\right]+O\left(n^{-1}\right), \quad\left(\gamma_{2} k_{2} E I>1\right) \\
\frac{1}{2 \gamma_{2} l}\left[\ln \left|\frac{\gamma_{2} k_{2} E I-1}{\gamma_{2} k_{2} E I+1}\right|+(2 n+1) \pi i\right]+O\left(n^{-1}\right), \quad\left(\gamma_{2} k_{2} E I<1\right)
\end{array}\right.
\end{aligned}
$$

Proof: Let us consider the asymptotic analysis of the eigenvalues using the procedure developed in [28]. First we set $\Phi(x) \equiv\left[\varphi_{1}(x), \varphi_{1}^{\prime}(x), \varphi_{3}(x), \varphi_{3}^{\prime}(x)\right]^{T}$. Then (26) can be rewritten as follows: $U^{D}(s, x) \Phi(x) \equiv \Phi^{\prime}(x)-M(s) \Phi(x)=0, U^{B}(s) \Phi \equiv Q^{0}(s) \Phi(0)$ $+Q^{l}(s) \Phi(l)=0$, where $M(s), Q^{0}(s), Q^{l}(s) \in \mathrm{C}^{4 \times 4}$ are the matrixes. Now we introduce new variable

$$
P(s)=\left(\begin{array}{cc}
P_{1}(s) & 0_{2 \times 2} \\
0_{2 \times 2} & P_{2}(s)
\end{array}\right), \quad P_{i}(s)=\left(\begin{array}{cc}
\gamma_{i} s & \gamma_{i} s \\
\gamma_{i}^{2} s^{2} & -\gamma_{i}^{2} s^{2}
\end{array}\right),
$$

and define the following: $\Psi(x) \equiv P^{-1}(s) \Phi(x)$ and $\hat{U}^{D}(s, x) \equiv$ $P^{-1}(s) U^{D}(s, x) P(s)$. We then obtain

$\hat{U}^{D}(s, x) \Psi(x)=\Psi^{\prime}(x)-\hat{M}(s) \Psi(x)=0$.

In particular, the matrix $\hat{M}(s)$ can be expanded to $\hat{M}(s)=s \hat{M}_{1}+\hat{M}_{0}+s^{-1} \hat{M}_{-1}$, where $\hat{M}_{i}(s) \in \mathrm{C}^{4 \times 4}, i=-1,0,1$. A fundamental matrix solution to (29) for a sufficiently large $s$ has already been obtained in [28]:

$\Psi(x)=\left(\hat{\Theta}_{0}(x)+\frac{\hat{\Theta}_{1}(x)}{s}+\frac{\hat{\Theta}(x, s)}{s^{2}}\right) E(s, x)$,

where $\hat{\Theta}(x, s)=\hat{\Theta}_{2}(x)+\hat{\Theta}_{3}(x) / s+\cdots$ is uniformly bounded, and $E(s, x)=\operatorname{diag}\left(\mathrm{e}^{\gamma_{1} s x}, \mathrm{e}^{-\gamma_{1} s x}, \mathrm{e}^{\gamma_{2} s x}, \mathrm{e}^{-\gamma_{2} s x}\right)$. The specific values of $\hat{\Theta}_{0}(x), \hat{\Theta}_{1}(x), \hat{\Theta}_{2}(x), \cdots$ can be derived by substituting (30) into (29), and by using the assumption $\gamma_{1} \neq \gamma_{2}$.

A fundamental solution $\Phi(x)$ to the original eigenvalue problem is $\Phi(x)=P(s) \Psi(x)$. Calculating the characteristic equation while considering this fact leads to

$\Delta=\operatorname{det}\left(U^{B}(s) \Phi\right)=\gamma_{1}^{2} \gamma_{2}^{3} s^{7} \Delta_{1} \Delta_{2}=0, \quad \Delta_{1}=\mathrm{e}^{-\gamma_{1} s l}-\mathrm{e}^{\gamma_{1} s l}+O\left(s^{-1}\right)$,

$\Delta_{2}=\left(1+\gamma_{2} k_{2} E I\right) \mathrm{e}^{\gamma_{2} s l}+\left(1-\gamma_{2} k_{2} E I\right) \mathrm{e}^{-\gamma_{2} s l}+O\left(s^{-1}\right)$.

Here, we can solve $\Delta_{1}=0$ using Rouche's theorem [28] and we obtain (27). On the other hand, using the same procedure, $\Delta_{2}=0$ gives (28). From these, the spectrum $\sigma(A)$ is simple, with sufficiently large moduli, and has two asymptotic branches (27) and (28).

In Lemma 3 we assumed that $\gamma_{2} k_{2} E I \neq 1$ and $\gamma_{1} \neq \gamma_{2}$. If we do not assume $\gamma_{2} k_{2} E I \neq 1$, the asymptotic branch (28) vanishes, and the spectrum $\sigma(A)$ has only one branch (27). On the other hand, it is known that the assumption $\gamma_{1} \neq \gamma_{2}$ holds for physical systems; i.e., the equality $\gamma_{1}=\gamma_{2}$ has no meaning from the physical point of view [29].

Now we obtain the following theorem for non-exponential stability of the closed-loop system. 
Theorem 2: If the feedback gain $k_{i}, i=1, \ldots, 4$, satisfies (21) and $\gamma_{1} \neq \gamma_{2}$ is satisfied, then the closed-loop system (20) is non-exponentially stable.

Proof: Let $T(t)$ be a $C_{0}$-semigroup of contractions generated by the operator $A$. For the growth bound of $T(t), \omega_{0}=$ $\inf _{t>0} \log \|T(t)\| / t$, and the spectral bound of the operator $A$, $s(A)=\sup _{s \in \sigma(A)} \operatorname{Re}(s)$, it is known that the inequality $s(A) \leq \omega_{0}$ always holds [30]. On the other hand, from Lemma 3, $s(A)=0$ and the growth bound $\omega_{0}$ does not become negative. Therefore the closed-loop system (20) cannot be exponentially stable.

From Theorem 1 and 2, we could show that our previously proposed force controller [14], [17] realized the force control of the flexible Timoshenko arm constrained to a rigid environment. In addition, the controller asymptotically stabilized the closed-loop system, but could not exponentially stabilize it. In contrast to the dynamics of the Euler-Bernoulli beam, the Timoshenko beam has the dynamics of transverse displacement (1), and the dynamics of the rotation of the cross section (2). To correspond with these two dynamics, the eigenvalues of the operator $A$ has two asymptotic branches. (Note that there are no eigenvalues by the dynamics of the motor at a place far enough away from the origin.) Although one of the two branches, (28), is away from the imaginary axis, the remaining branch, (27), approaches the imaginary axis, and thus the closed-loop system cannot be exponentially stable. Therefore we can declare that the controller (9) is not sufficient to control the dynamics of the transverse displacement and of the rotation of the cross section at the same time. To overcome this problem, we consider the flexible Timoshenko arm making contact with a soft object. By utilizing the damping force of the soft object, as well as the controller, we try to realize the exponential stability of the closed-loop system.

Here, note that there have been many studies about the polynomial stability of Timoshenko beams [8], [27]; these, however, focused on the vibration-suppression of the Timoshenko beam, and not on the contact-force control problem of the flexible Timoshenko arm. Although we attempted to show the polynomial stability of the closed-loop system, we could not prove it. We consider this to be a problem for future research.

\section{FLEXIBLE TIMOSHENKO ARM WITH A SOFT ENVIRONMENT}

\section{A. A flexible Timoshenko arm with a soft environment}

Fig. 2 shows a one-link flexible Timoshenko arm constrained with a soft environment. To represent the soft object, we use the translational spring and damper, and the rotational damper. That is, we assume that one end of the arm is fixed to the control motor, and the other end is connected to the environment through the spring and dampers. The difference between Figs. 1 and 2 is the boundary at the tip of the arm. Since the tip makes contact with the surface of the object through the spring and dampers, the geometric constraint $\Lambda=0$

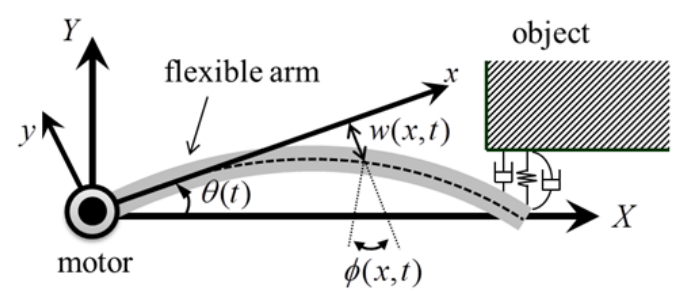

Fig. 2. Flexible Timoshenko arm making contact with a soft object.

vanishes. Further, due to the spring and the dampers, we need to consider the potential energy $k_{e}[l \theta(t)+w(l, t)]^{2} / 2$ and the dissipation energy $d_{e}[l \dot{\theta}(t)+\dot{w}(l, t)]^{2} / 2+c_{e} \dot{\phi}^{2}(l, t) / 2$, where $k_{e}$, $d_{e}$, and $c_{e}$ are the spring coefficient of the translational spring, the damping coefficient of the translational damper, and the damping coefficient of the rotational damper, respectively. Using Hamilton's principle and the Rayleigh dissipation function, we arrive at the following equations of motion of the system in Fig. 2: for $x \in(0, l)$ and $t \geq 0$

$$
\begin{aligned}
& \rho_{1}[\ddot{w}(x, t)+x \ddot{\theta}(t)]+K\left[\phi^{\prime}(x, t)-w^{\prime \prime}(x, t)\right]=0, \\
& \rho_{2}[\ddot{\phi}(x, t)+\ddot{\theta}(t)]+K\left[\phi(x, t)-w^{\prime}(x, t)\right]-E I \phi^{\prime \prime}(x, t)=0, \\
& w(0, t)=\phi(0, t)=0, \\
& E I \phi^{\prime}(l, t)=-c_{e} \dot{\phi}(l, t), \\
& K\left[\phi(l, t)-w^{\prime}(l, t)\right]=k_{e}[l \theta(t)+w(l, t)]+d_{e}[l \dot{\theta}(t)+\dot{w}(l, t)], \\
& J \ddot{\theta}(t)=\tau_{m}(t)+E I \phi^{\prime}(0, t) \equiv \tau(t) .
\end{aligned}
$$

Here, note that the contact force becomes:

$$
\lambda(t)=-k_{e}[l \theta(t)+w(l, t)]-d_{e}[l \dot{\theta}(t)+\dot{w}(l, t)] .
$$

\section{B. Control objective}

At the desired equilibrium point $\left(\lambda(t)=\lambda_{d}, \dot{w}(x, t)=\dot{\phi}(x, t)=\dot{\theta}(t)=\right.$ 0 ), the static transverse displacement $w_{d}(x)$, static rotation of the cross section $\phi_{d}(x)$, and static angle of the motor $\theta_{d}$ are related as follows:

$$
\left\{\begin{array}{l}
w_{d}(x)=\lambda_{d} x\left(\frac{1}{K}+\frac{l x}{2 E I}-\frac{x^{2}}{6 E I}\right), \quad \phi_{d}(x)=\frac{\lambda_{d} x}{E I}\left(l-\frac{x}{2}\right), \\
\theta_{d}=-\lambda_{d}\left(\frac{1}{l k_{e}}+\frac{1}{K}+\frac{l^{2}}{3 E I}\right) .
\end{array}\right.
$$

Here, $\theta_{d}$ is affected by the translational spring.

\section{Semigroup setting}

Using the variables (10) and (38), and using the controller (9), the equations of motion under the soft object can be rewritten as follows: for $x \in(0, l)$ and $t \geq 0$

$$
\begin{aligned}
& \rho_{1} \ddot{y}_{1}(x, t)+K\left[y_{2}^{\prime}(x, t)-y_{1}^{\prime \prime}(x, t)\right]=0, x \in(0, l), \quad t \geq 0, \\
& \rho_{2} \ddot{y}_{2}(x, t)+K\left[y_{2}(x, t)-y_{1}^{\prime}(x, t)\right]-E I y_{2}^{\prime \prime}(x, t)=0, \\
& y_{1}(0, t)=0, \quad E I y_{2}^{\prime}(l, t)=-c_{e} \dot{y}_{2}(l, t), \\
& K\left[y_{2}(l, t)-y_{1}^{\prime}(l, t)\right]=k_{e} y_{1}(l, t)+d_{e} \dot{y}_{1}(l, t), \\
& \dot{\eta}(t)=-k_{1} E I y_{2}^{\prime}(0, t)+k_{3} y_{2}(0, t)+D \dot{y}_{2}(0, t) .
\end{aligned}
$$

The differences between the equations of motion (11)-(14) and (39)-(43) are the boundary conditions at $x=l$. Since the boundary conditions are different, the state space, the inner product, and the operator of the system are slightly altered.

As the state space, we introduce the following Hilbert space: 
$H_{2}=\widetilde{H}_{0}^{1}(0, l) \times L^{2}(0, l) \times H^{1}(0, l) \times L^{2}(0, l) \times \mathrm{C}^{2}$,

where $\widetilde{H}_{0}^{m}(0, l)=\left\{u \in H^{m}: u(0)=0\right\}$. Defining the inner product

$$
\begin{aligned}
& \langle z, \hat{z}\rangle_{H_{2}}=\frac{\rho_{1}}{2} \int_{0}^{l} v_{1} \overline{\hat{v}_{1}} \mathrm{~d} x+\frac{\rho_{2}}{2} \int_{0}^{l} v_{2} \overline{\hat{v}_{2}} \mathrm{~d} x+\frac{E I}{2} \int_{0}^{l} u_{2}^{\prime} \overline{\hat{u}_{2}^{\prime}} \mathrm{d} x+\frac{k_{e}}{2} u_{1}(l) \overline{\hat{u}_{1}(l)} \\
& \quad+\frac{K}{2} \int_{0}^{l}\left(u_{2}-u_{1}^{\prime}\right) \overline{\left(\hat{\hat{u}_{2}}-\hat{u}_{1}^{\prime}\right)} \mathrm{d} x+\frac{k_{3}}{2 k_{1}} u_{2}(0) \overline{\hat{u}_{2}(0)}+\frac{1}{2\left(k_{1}+k_{2} D\right)} \eta \overline{\hat{\eta}},
\end{aligned}
$$

for $z=\left(u_{1}, v_{1}, u_{2}, v_{2}, \eta\right)^{T}$ and $\hat{z}=\left(\hat{u}_{1}, \hat{v}_{1}, \hat{u}_{2}, \hat{v}_{2}, \hat{\eta}\right)^{T} \in H_{2}$. Here we assumed that $k_{1}+k_{2} D>0$, which holds if $D>0$. The state space $H_{2}$, together with (45), becomes a Hilbert space. Further, we also define a linear operator $B: D(B) \subset H_{2} \rightarrow H_{2}$ by

$$
\begin{gathered}
B z=\left[v_{1}, \frac{K}{\rho_{1}}\left(u_{1}^{\prime \prime}-u_{2}^{\prime}\right), v_{2}, \frac{K}{\rho_{2}}\left(u_{1}^{\prime}-u_{2}\right)+\frac{E I}{\rho_{2}} u_{2}^{\prime \prime},\right. \\
\left.-k_{1} E I u_{2}^{\prime}(0)+k_{3} u_{2}(0)+D v_{2}(0)\right]^{T} \\
D(B)=\left\{z \in \widetilde{H}_{0}^{2}(0, l) \times \widetilde{H}_{0}^{1}(0, l) \times H^{2}(0, l) \times H^{1}(0, l) \times \mathrm{C}:\right. \\
E I u_{2}^{\prime}(l)=-c_{e} v_{2}(l), K\left[u_{2}(l)-u_{1}^{\prime}(l)\right]=k_{e} u_{1}(l)+d_{e} v_{1}(l), \\
\left.\eta=k_{2} E I u_{2}^{\prime}(0)-v_{2}(0)-k_{2} k_{3} u_{2}(0) / k_{1}\right\}
\end{gathered}
$$

The closed-loop system (39)-(43) can then be written as a first order evolution equation on $\mathrm{H}_{2}$,

$\dot{z}(t)=B z(t), \quad z(0)=z_{0}$,

where $z(t)=\left(y_{1}(\cdot, t), \dot{y}_{1}(\cdot, t), y_{2}(\cdot, t), \dot{y}_{2}(\cdot, t), \eta(t)\right)^{T}$. As the properties of the new closed-loop system, we obtain the following lemma:

Lemma 4: If the feedback gain $k_{i}, i=1, \ldots, 4$, satisfies (21), then the operator $B$ generates a $C_{0}$-semigroup of contractions. Further, the operator $B^{-1}$ is compact. Therefore, the spectrum $\sigma(B)$ of the operator $B$ consists only of the isolated eigenvalues.

Proof: We show that the operator $B$ is dissipative. For any $z=\left(u_{1}, v_{1}, u_{2}, v_{2}, \eta\right)^{T} \in D(B)$, it follows that

$$
\begin{aligned}
2 \operatorname{Re}\langle B z, z\rangle_{H_{2}}= & -d_{e}\left|v_{1}(l)\right|^{2}-c_{e}\left|v_{2}(l)\right|^{2}-\frac{D}{k_{1}+k_{2} D}\left|v_{2}(0)\right|^{2} \\
& -\frac{k_{2}}{k_{1}\left(k_{1}+k_{2} D\right)}\left|k_{1} E I u_{2}^{\prime}(0)-k_{3} u_{2}(0)\right|^{2} \leq 0 .
\end{aligned}
$$

Hence, the operator $B$ is dissipative. The facts that $0 \in \rho(B)$ and $B^{-1}$ is compact are straightforward, and we easily obtain them using the same procedure as in Lemma 1; thus we omit them here. From these facts, the proof is completed

\section{EXPONENTIAL STABILITY}

Although we tried to show the exponential stability of the closed-loop system using the energy-Lyapunov method, we could not find the appropriate Lyapunov functional. Instead, we investigate the exponential stability using the frequency domain method. According to this method, we need to show the following two facts to prove exponential stability [23]:

(i) $\rho(B) \supset\{i \beta: \beta \in \mathrm{R}\} \equiv i \mathrm{R}$,

(ii) $\varlimsup_{|\beta| \rightarrow \infty}\left\|(\mathrm{i} \beta-B)^{-1}\right\|_{H^{2}}<\infty$.

Below, we show fact (i) in Lemma 5, and (ii) in Lemma 6.
Lemma 5: Assume that the feedback gain $k_{i}, i=1, \ldots, 4$, satisfies (21). Then, $i \mathrm{R} \subset \rho(B)$.

Proof: We have shown that the spectrum $\sigma(B)$ consists only of the isolated eigenvalues in Lemma 4. Thus, to prove that the imaginary axis belongs to the resolvent set $\rho(B)$, we show that there are no eigenvalues on the imaginary axis.

Let $s=i \beta$ and $\varphi=\left[\varphi_{1}, \varphi_{2}, \varphi_{3}, \varphi_{4}, \varphi_{5}\right]^{T} \in D(B)$ be an eigenvalue and the eigenfunction of the operator $B$, respectively, where $\beta \in \mathrm{R}$. Now let us consider the eigenvalue problem $B \varphi=s \varphi$. Here, we have shown that $0 \in \rho(B)$, and thus $\beta \neq 0$. Then, we can obtain $\operatorname{Re}\langle B \varphi, \varphi\rangle_{H_{2}}=0$, and this means:

$\varphi_{2}(l)=\varphi_{4}(0)=\varphi_{4}(l)=0, \quad k_{1} E I \varphi_{3}^{\prime}(0)-k_{3} \varphi_{3}(0)=0$.

Eliminating $\varphi_{2}, \varphi_{4}$, and $\varphi_{5}$ in the equation $B \varphi=s \varphi$, and using (52) leads to (24) with $\varphi_{3}(l)=0$. Therefore, from Lemma 2, the eigenvalue problem $B \varphi=s \varphi$ has only a zero solution, and thus the proof is complete.

Lemma 6: Assume that the feedback gain $k_{i}, i=1, \ldots, 4$, satisfies (21). Then, (51) holds.

Proof: According to the contradiction argument method [23], if (51) is false, then there exists a sequence $\beta_{n} \in \mathrm{R}$ with $\beta_{n} \rightarrow \infty$, and a sequence $z_{n} \in D(B)$ with $\left\|z_{n}\right\|_{H_{2}}=1$ such that:

$\left(i \beta_{n}-B\right) z_{n} \equiv \varphi_{n} \rightarrow 0$ in $H_{2}$,

where $z=\left(u_{1 n}, v_{1 n}, u_{2 n}, v_{2 n}, \eta_{n}\right)^{T}$, and $\varphi_{n}=\left(\varphi_{1 n}, \varphi_{2 n}, \varphi_{3 n}, \varphi_{4 n}, \varphi_{5 n}\right)^{T}$. Here, (53) leads to the following:

$i \beta_{n} u_{1 n}-v_{1 n}=\varphi_{1 n}, \quad i \beta_{n} v_{1 n}-K\left(u_{1 n}^{\prime \prime}-u_{2 n}^{\prime}\right) / \rho_{1}=\varphi_{2 n}$,

$i \beta_{n} u_{2 n}-v_{2 n}=\varphi_{3 n}, \quad i \beta_{n} v_{2 n}-K\left(u_{1 n}^{\prime}-u_{2 n}\right) / \rho_{2}-E I u_{2 n}^{\prime \prime} / \rho_{2}=\varphi_{4 n}$,

$i \beta_{n} \eta_{n}+k_{1} E I u_{2 n}^{\prime}(0)-k_{3} u_{2 n}(0)-D v_{2 n}(0)=\varphi_{5 n}$,

$u_{1 n}(0)=v_{1 n}(0)=0, \quad E I u_{2 n}^{\prime}(l)=-c_{e} v_{2 n}(l)$,

$K\left[u_{2 n}(l)-u_{1 n}^{\prime}(l)\right]=k_{e} u_{1 n}(l)+d_{e} v_{1 n}(l)$,

$\eta_{n}=k_{2} E I u_{2 n}^{\prime}(0)-v_{2 n}(0)-k_{2} k_{3} u_{2 n}(0) / k_{1}$.

Now we show the contradictions of $\left\|z_{n}\right\|_{H_{2}}=1$, i.e., $\left\|z_{n}\right\|_{H_{2}} \rightarrow 0$.

First, we derive the required estimations for the proof. From (53), we obtain $\operatorname{Re}\left\langle\left(i \beta_{n}-B\right) z_{n}, z_{n}\right\rangle_{H_{2}} \rightarrow 0$ and this means

$\left|v_{1 n}(l)\right| \rightarrow 0,\left|v_{2 n}(0)\right| \rightarrow 0,\left|v_{2 n}(l)\right| \rightarrow 0,\left|k_{1} E I u_{2 n}^{\prime}(0)-k_{3} u_{2 n}(0)\right| \rightarrow 0$,

Here, using (57), (59), and (60) leads to

$\left|\eta_{n}\right| \rightarrow 0, \quad\left|u_{2 n}^{\prime}(l)\right| \rightarrow 0$.

The followings are obtained from $\left\|\varphi_{n}\right\|_{H_{2}} \rightarrow 0$ :

$\left\{\left\|\varphi_{2 n}\right\|_{L^{2}} \rightarrow 0,\left\|\varphi_{3 n}^{\prime}\right\|_{L^{2}} \rightarrow 0,\left\|\varphi_{4 n}\right\|_{L^{2}} \rightarrow 0,\left\|\varphi_{3 n}-\varphi_{1 n}^{\prime}\right\|_{L^{2}} \rightarrow 0\right.$,

$\left\{\left|\varphi_{1 n}(l)\right| \rightarrow 0,\left|\varphi_{3 n}(0)\right| \rightarrow 0,\left|\varphi_{5 n}\right| \rightarrow 0\right.$.

In addition, (62) and (A1) give

$\left\|\varphi_{1 n}\right\|_{L^{2}} \rightarrow 0,\left\|\varphi_{1 n}^{\prime}\right\|_{L^{2}} \rightarrow 0,\left\|\varphi_{3 n}\right\|_{L^{2}} \rightarrow 0$.

From the first equation in (54), the first equation in (55), (60), (62), and the trace theorem, we have

$\left\{\begin{array}{l}\left|\beta_{n} u_{1 n}(l)\right| \rightarrow 0,\left|u_{1 n}(l)\right| \rightarrow 0,\left|u_{2 n}(0)\right| \rightarrow 0,\left|\varphi_{3 n}(l)\right| \rightarrow 0, \\ \left|\beta_{n} u_{2 n}(l)\right| \rightarrow 0,\left|u_{2 n}(l)\right| \rightarrow 0 .\end{array}\right.$ 
Further, using (58), (60), and (64), we obtain

$$
\left|u_{1 n}^{\prime}(l)\right| \rightarrow 0 \text {. }
$$

Next, combining the two equations in (54), multiplying the obtained equation by $x \overline{u_{1 n}^{\prime}}$, and integrating it yields

$$
\int_{0}^{l}\left[\rho_{1} \beta_{n}^{2} u_{1 n}-K\left(u_{2 n}^{\prime}-u_{1 n}^{\prime \prime}\right)\right] x \overline{u_{1 n}^{\prime}} \mathrm{d} x=-\rho_{1} \int_{0}^{l}\left(i \beta_{n} \varphi_{1 n}+\varphi_{2 n}\right) x \overline{u_{1 n}^{\prime}} \mathrm{d} x .
$$

Here, a simple calculation using (57), (63), (64) and the boundedness of $\left\|z_{n}\right\|_{H_{2}}$, that is $\left\|z_{n}\right\|_{H_{2}}=1$, in (66) gives $\rho_{1} \int_{0}^{l} \beta_{n}^{2} u_{1 n} x \overline{u_{1 n}^{\prime}} \mathrm{d} x+K \int_{0}^{l} K\left(u_{1 n}^{\prime \prime}-u_{2 n}^{\prime}\right) x \overline{u_{1 n}^{\prime}} \mathrm{d} x \rightarrow 0$. Further, using (64) and (65) in this equation, and taking the real part of the obtained equation, we obtain:

$$
-\frac{\rho_{1}}{2}\left\|\beta_{n} u_{1 n}\right\|_{L^{2}}^{2}-\frac{K}{2}\left\|u_{1 n}^{\prime}\right\|_{L^{2}}^{2}-K \operatorname{Re} \int_{0}^{l} x u_{2 n}^{\prime} \overline{u_{1 n}^{\prime}} \mathrm{d} x \rightarrow 0 .
$$

Similarly, if we combine the two equations in (55), multiply the obtained equation by $x \overline{u_{2 n}^{\prime}}$, and integrate it, then the calculations using (61), (63), (64), and the boundedness of $\left\|z_{n}\right\|_{H_{2}}$ lead to:

$-\frac{\rho_{2}}{2}\left\|\beta_{n} u_{2 n}\right\|_{L^{2}}^{2}+K \operatorname{Re} \int_{0}^{l} x u_{1 n}^{\prime} \overline{u_{2 n}^{\prime}} \mathrm{d} x+\frac{K}{2}\left\|u_{2 n}\right\|_{L^{2}}^{2}-\frac{E I}{2}\left\|u_{2 n}^{\prime}\right\|_{L^{2}}^{2} \rightarrow 0$.

By taking the sum of (67) and (68), we obtain $\rho_{1}\left\|\beta_{n} u_{1 n}\right\|_{L^{2}}^{2}+$ $K\left\|u_{1 n}^{\prime}\right\|_{L^{2}}^{2}+\left(\rho_{2}-K / \beta_{n}^{2}\right)\left\|\beta_{n} u_{2 n}\right\|_{L^{2}}^{2}+E I\left\|u_{2 n}^{\prime}\right\|_{L^{2}}^{2} \rightarrow 0$. Here, each coefficient is positive, and thus we obtain

$\left\|\beta_{n} u_{i n}\right\|_{L^{2}} \rightarrow 0,\left\|u_{i n}\right\|_{L^{2}} \rightarrow 0,\left\|u_{i n}^{\prime}\right\|_{L^{2}} \rightarrow 0, \quad i=1,2$

In addition, we also obtain the following from the first equations in (54) and (55), and (63) and (69):

$\left\|v_{\text {in }}\right\|_{L^{2}} \rightarrow 0, \quad i=1,2$.

Finally, using (61), (64), (69), (70), and noting that $K / 2\left\|u_{2 n}-u_{1 n}^{\prime}\right\|_{L^{2}}^{2} \leq K\left\|u_{2 n}\right\|_{L^{2}}^{2}+K\left\|u_{1 n}^{\prime}\right\|_{L^{2}}^{2}$ because of the inequality

$|a+b|^{2} \leq(|a|+|b|)^{2} \leq 2\left(|a|^{2}+|b|^{2}\right), \quad a, b \in \mathrm{C}$,

we obtain $\left\|z_{n}\right\|_{H_{2}} \rightarrow 0$, and this is the contradiction of $\left\|z_{n}\right\|_{H_{2}}=1$. Thus, the claim is proved.

Lemma 5 and 6 are summarized in the following theorem for the exponential stability of the closed-loop system (48).

Theorem 3: Assume that the feedback gain $k_{i}, i=1, \ldots, 4$, satisfies (21). Then, the closed-loop system (48) is exponentially stable.

Proof: Lemma 5, 6, and the frequency domain method [23] leads to the exponential stability of the closed-loop system.

\section{Simulations}

We investigate the energy decay of the closed-loop system with both a rigid and a soft environment. To avoid numerical errors, we use the following small parameters in the simulations: $\rho_{1}=0.8^{2}, \rho_{2}=0.5^{2}$, and $E I=K=l=1$. In addition, we set $\lambda_{d}=-5$, and use $k_{1}=4, k_{2}=0.1, k_{3}=2$, and $k_{4}=0.1$ for the rigid environment, and $k_{1}=4, k_{2}=0.1, k_{3}=2, k_{4}=0.1, k_{e}=300$, and $d_{e}=c_{e}=0.1$ for the soft environment. The numerical simulation is conducted by the finite difference method, and we use $\Delta x=$ 0.0125 and $\Delta t=0.001$ for the mesh of the spatial variable and the time variable, respectively.

Fig. 3 shows the simulation results. Here, we use $E_{1}=\|z\|_{H}^{2}$ as the energy for the rigid environment, and $E_{2}=\|z\|_{H_{2}}^{2}$ for the soft environment. In Fig. 3 (a), the solid line represents $E_{2}$, and the dotted line $E_{1}$. Both energies converge to zero, and we see that convergence speed in the soft environment is faster than that in the rigid environment. The soft environment has the damping forces of the soft object in addition to the controller, so that the fast convergence speed is expected.

On the other hand, we also carried out numerical simulations to investigate whether the condition (21) is necessary for stability. For this purpose, we consider the same simulation described above in the following four cases: (i) $k_{1}$ is changed (we considered four $k_{1}: k_{1}=2,1,0.5,0.14$ ) while $k_{2}=0.1, k_{3}=2$, and $k_{4}=0.1$; (ii) $k_{2}$ is changed (four $k_{2}: k_{2}=0.2,1,2,2.9$ ) while $k_{1}=4, k_{3}=2$, and $k_{4}=0.1$; (iii) $k_{3}$ is changed (three $k_{3}$ : $k_{3}=10,11,15$ for the rigid environment case and $k_{3}=11,15,22$ for the soft environment case) while $k_{1}=4, k_{2}=0.1$, and $k_{4}=0.1$; and (iv) $k_{4}$ is changed (four $k_{4}: k_{4}=0.05,0.01,0.005,0.001$ ) while $k_{1}=4, k_{2}=0.1$, and $k_{3}=2$. Here, the changing ranges of the gains are set so that $k_{1}+k_{2} D>0$ and $D \leq 0$ are satisfied. Here note that $k_{1}+k_{2} D>0$ is required for $E_{1}$ and $E_{2}$ to be well defined. As an example, we show the results of case (iii) in Fig. 3 (b) and (c). Fig. 3 (b) shows $E_{2}$ and (c) shows $E_{1}$. We can see $E_{1}$ diverges when $k_{3}$ is larger than 11 . On the other hand, $E_{2}$ diverges when $k_{3}$ is larger than 15 . Thus both cases have stable situation even if we set the feedback gains so that $D<0$. In particular, we also confirmed that the soft environment had a slightly wider stable range than the rigid environment. On the other hand, in cases (i) and (iv), the convergence speed of the energy is slow when $k_{1}$ and $k_{4}$ are small, but the energy does not diverge in either the case of the soft or rigid environment. Further, in case (ii), the convergence speed of the energy is high when $k_{2}$ is large; again, the energy does not diverge in either case. Therefore, from this point of view, we could confirm the possibility that stability would be maintained even if the condition (21) does not hold.

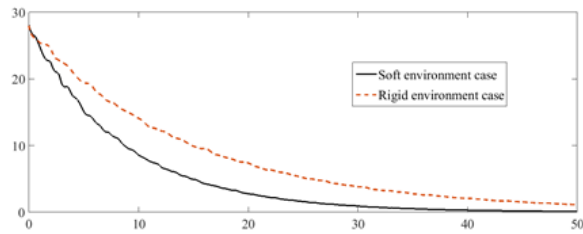

(a) Energy decays

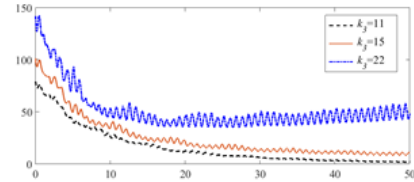
environment case (b) Influences of $k_{3}$ in Soft

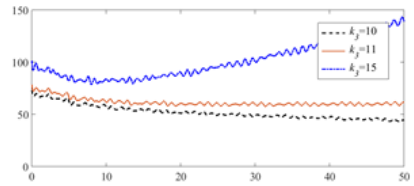

(c) Influences of $k_{3}$ in Rigid environment case 


\section{CONCLUSION}

The aim of this paper was to realize contact-force control of a one-link flexible Timoshenko arm. To realize this aim, we first applied our previously proposed force controller to the problem of the flexible Timoshenko arm making contact with a rigid environment, and realized force control of a one-link flexible Timoshenko arm. At the same time, we also showed that the closed-loop system could not be exponentially stable. To overcome this problem, we considered the flexible Timoshenko arm making contact with a soft object. By utilizing the damping force of the soft object, as well as the controller, we realized exponential stability of the closed-loop system. Furthermore, we investigated the energy decay of the closed-loop system under the rigid and soft environments, and investigated the differences between these systems. The Timoshenko beam can be used in a wider application than the Euler-Bernoulli beam, and thus its force control is a generally important issue in order for the flexible arm to be useful for more complex tasks.

\section{APPENDIX}

We show the following estimate, which is used in the proof of Lemma 6:

$\left\|\left(u_{1}, u_{2}\right)\right\|_{2}^{2} \leq \gamma_{1}\left\|\left(u_{1}, u_{2}\right)\right\|_{1}^{2}$,

for a positive constant $\gamma_{1}$, where

$$
\begin{aligned}
& 2\left\|\left(u_{1}, u_{2}\right)\right\|_{1}^{2}=E I\left\|u_{2}^{\prime}\right\|_{L^{2}}^{2}+K\left\|u_{2}-u_{1}^{\prime}\right\|_{L^{2}}^{2}+k_{3}\left|u_{2}(0)\right|^{2} / k_{1}, \\
& \left\|\left(u_{1}, u_{2}\right)\right\|_{2}^{2}=\left\|u_{1}\right\|_{L^{2}}^{2}+\left\|u_{1}^{\prime}\right\|_{L^{2}}^{2}+\left\|u_{2}\right\|_{L^{2}}^{2}+\left\|u_{2}^{\prime}\right\|_{L^{2}}^{2},
\end{aligned}
$$

for $\left(u_{1}, u_{2}\right) \in H_{0}^{1}(0, l) \times H^{1}(0, l)$. Here note that the following discussion is also valid for $\left(u_{1}, u_{2}\right) \in \widetilde{H}_{0}^{1}(0, l) \times H^{1}(0, l)$.

From the equations $u_{i}(x)=\int_{0}^{x} u_{i}^{\prime}(x) \mathrm{d} x+u_{i}(0)$ for $i=1,2$, the Cauchy-Schwartz inequality and inequality (71), we obtain $\left\|u_{i}\right\|_{L^{2}}^{2} \leq \gamma_{2}\left[\left\|u_{i}^{\prime}(x)\right\|_{L^{2}}^{2}+\left|u_{i}(0)\right|^{2}\right]$, where $\gamma_{2}$ is a positive constant. On the other hand, integrating the equation $\left|u_{2}-u_{1}^{\prime}\right|^{2}=\left|u_{2}\right|^{2}-2 \operatorname{Re}\left(u_{2} \overline{u_{1}^{\prime}}\right)+\left|u_{1}^{\prime}\right|^{2}$ and using the inequality $2|a||b| \leq \delta|a|^{2}+|b|^{2} / \delta$ for $a, b \in \mathrm{C}, \delta \in \mathrm{R}, \delta>0$, gives $\left\|u_{2}-u_{1}^{\prime}\right\|_{L^{2}}^{2} \geq(1-\delta)\left\|u_{2}\right\|_{L^{2}}^{2}+(1-1 / \delta)\left\|u_{1}^{\prime}\right\|_{L^{2}}^{2}$. Therefore, using this estimate and (A4), we obtain

$\left\|\left(u_{1}, u_{2}\right)\right\|_{1}^{2} \geq \gamma_{3}\left[\left\|u_{2}\right\|_{L^{2}}^{2}+\left\|u_{1}^{\prime}\right\|_{L^{2}}^{2}\right]$,

where $\gamma_{3}=\min \left\{\gamma_{4}, \gamma_{5}\right\} \quad, \quad \gamma_{4}=\min \left\{E I / 2, k_{3} /\left(2 k_{1}\right)\right\} / \gamma_{2}+K(1-\delta) / 2$, $\gamma_{5}=K(1-1 / \delta) / 2$, and we set $1<\delta<1+2 \min \left\{E I / 2, k_{3} /\left(2 k_{1}\right)\right\} /\left(K \gamma_{2}\right)$. Thus, we obtain (A1) by using (A4) and (A5).

\section{REFERENCES}

[1] Ö. Morgül, "Dynamic Boundary Control of the Timoshenko Beam," Automatica, vol.28, no.6, pp.1255-1260, 1992

[2] F. Zhang, D.M. Dawson, M.S. de Queiroz, and P. Vedagarbha, "Boundary Control of the Timoshenko Beam with Free-End Mass/Inertial Dynamics," In Proc. IEEE CDC, pp.245-250, 1997.

[3] S.W. Taylor and S.C.B. Yau, "Boundary control of a rotating Timoshenko beam," ANZIAM J., vol.44, pp.E143-E184, 2003.

[4] M. Grobbelaar-Van Dalsen, "Uniform stability for the Timoshenko beam with tip load," J. Math. Anal. Appl., vol.361, no.2, pp.392-400, 2010.
[5] Z.J. Han and G.Q. Xu, "Dynamical behavior of a hybrid system of nonhomogeneous timoshenko beam with partial non-collocated inputs," $J$. Dyn. Control Syst., vol.17, no.1, pp.77-121, 2011.

[6] W. He, S. Zhang, and S.S. Ge, "Boundary Output-Feedback Stabilization of a Timoshenko Beam Using Disturbance Observer," IEEE Trans. Ind. Electron., vol.60, no.11, pp.5186-5194, 2013.

[7] H. Ramírez, Y. Le Gorrec, A. Macchelli, and H. Zwart, "Exponential Stabilization of Boundary Controlled Port-Hamiltonian Systems With Dynamic Feedback," IEEE Trans. Autom. Control, vol.59, no.10, pp.2849-2855, 2014.

[8] J. E. Muñoz Rivera and A. I. Ávila, "Rates of decay to non homogeneous Timoshenko model with tip body," J. Differential Equations, vol.258, no. 10, pp.3468-3490, 2015

[9] T. Yoshikawa, "Force control of robot manipulators," In Proc. of IEEE ICRA, pp.220-226, 2000.

[10] L. Villani and J. De Schutter, "Force Control," in Handbook of Robotics, B. Siciliano and O. Khatib, eds. New York: Springer, pp.161-185, ch. 7, 2008.

[11] Y. Morita, F. Matsuno, Y. Kobayashi, M. Ikeda, H. Ukai, and H. Kando, "Lyapunov-based force control of a flexible arm considering bending and torsional deformation," In Proc. of IFAC World Congress, pp.832-832, 2002.

[12] F.M. Ching and D. Wang, "Exact solution and infinite-dimensional stability analysis of a single flexible link in collision," IEEE Trans. Robot. Autom. Vol.19, no.6, pp.1015-1020, 2003.

[13] Y. Shen, N. Xi, U.C. Wejinya, W.J. Li, and J. Xiao, "Infinite dimensional system approach for hybrid force/position control in micromanipulation," In Proc. of IEEE ICRA, pp.2912-2917, 2004.

[14] T. Endo, F. Matsuno, and H. Kawasaki, "Force Control and Exponential Stabilisation of One-Link Flexible Arm," Int. J. Control, vol.87, no.9, pp.1794-1807, 2014.

[15] T. Endo and H. Kawasaki, "Bending Moment-based Force Control of Flexible Arm Under Gravity," Mechanism and Machine Theory, vol.79, pp.217-229, 2014.

[16] F. Matsuno, A. Hayashi, "PDS cooperative control of two one-link flexible arms," In Proc. of IEEE ICRA, pp.1490-1495, 2000.

[17] T. Endo, F. Matsuno, and H. Kawasaki, "Simple boundary cooperative control of two one-link flexible arms for grasping," IEEE Trans. Autom. Control, vol.54, no.10, pp.2470-2476, 2009.

[18] H. Doua and S. Wang, "A boundary control for motion synchronization of a two-manipulator system with a flexible beam," Automatica, vol.50, no.12, pp.3088-3099, 2014.

[19] T.C. Huang, "The Effect of Rotary Inertia and of Shear Deformation on the Frequency and Normal Mode Equations of Uniform Beams With Simple End Conditions," J. Appl. Mech., vol.28, no.4, pp.579-584, 1961.

[20] S.M. Han, H. Benaroya, and T. Wei, "Dynamics of transversely vibrating beams using four engineering theories," J. of Sound and Vibration, vol.225, no.5, pp.935-988, 1999.

[21] C. Guiver and M.R. Opmeer, "Non-dissipative boundary feedback for Rayleigh and Timoshenko beams," Systems \& Control Letters, vol.59, no.9 pp. $578-586,2010$

[22] A. Bazaei and M. Moallem, "Improving Force Control Bandwidth of Flexible-Link Arms Through Output Redefinition," IEEE/ASME Trans. Mechatronics, vol.16, no.2 pp.380-386, 2011

[23] Z. Liu and S. Zheng, Semigroups Associated with Dissipative Systems, Chapman and Hall/CRC, 1999.

[24] A. Pazy, Semigroups of linear operators and applications to partial differential equations, Springer-Verlag, 1983.

[25] Z.J. Han and G.Q. Xu, "Stabilization and Riesz Basis Property of Two Serially Connected Timoshenko Beams System," Z. Angew. Math. Mech., vol.89, no.12, pp.962-980, 2009.

[26] W. Arendt and J.K. Batty, "Tauberian theorems and stability of one-parameter semigroups," Trans. the American Mathematical Society, vol.306, no. 2 pp.837-852, 1988.

[27] M. Bassam, D. Mercier, S. Nicaise, and A. Wehbe, "Polynomial stability of the Timoshenko system by one boundary damping," J. Math. Anal. Appl., vol.425, no.2 pp.1177-1203, 2015.

[28] G.Q. Xu, "Boundary feedback exponential stabilization of a Timoshenko beam with both ends free," Int. J. Control, vol.78, no.4 pp.286-297, 2005.

[29] Z.H. Liu and B.P. Rao, "Energy decay rate of the thermoelastic Bresse system," Z. Angew. Math. Phys., vol.60, no.1, pp.54-69, 2009.

[30] K.J. Engel and R. Nagel, One-Parameter Semigroups for Linear Evolution Equations, Springer-Verlag, 2000. 